\title{
Experimental Characterization of Weightlessness During Glider Parabolic Flights
}

\author{
Denis-Gabriel Caprace ${ }^{1,2}$ (D) $\cdot$ Camille Gontier ${ }^{1,3}$ (D) $\cdot$ Mohammad Iranmanesh ${ }^{1} \cdot$ Mehdi Scoubeau $^{1} \cdot$ Vladimir Pletser $^{4}$
}

Received: 16 April 2020 / Accepted: 24 September 2020 / Published online: 10 October 2020

(C) The Author(s) 2020

\begin{abstract}
Access to earthbound weightlessness is critical to many branches of applied sciences. Besides, several space systems require microgravity testing before their launch. Existing solutions (drop towers, parabolic flights, sounding rockets) offer variable durations and qualities of microgravity environment, but their cost and lead times make them unpractical for small actors such as universities or start-up companies. This leads to a growing interest for alternative microgravity platforms. Here, we study the use of gliders to perform parabolic flights at a lower cost, and we propose a systematic quantification of glider's 0-g flight capabilities. Results of our flight test campaign show that gliders offer up to $5.5 \mathrm{~s}$ of weightlessness, with excursions below $0.1 \mathrm{~g}$, and a satisfactory level of repeatability. Besides, the recordings do not suffer from the increased level of vibrations generated by piston engines, typical of light-aircraft-based alternatives. Operational considerations associated with glider parabolic flights are also discussed. Finally, we conclude that a microgravity platform based on gliders would be suitable especially for compact experiments and equipment in order to support accelerated design and development, or to produce preliminary experimental results.
\end{abstract}

Keywords Microgravity experiments · Gliders · Parabolic flights

\section{Introduction}

Experiments in a reduced-weight environment are a fundamental part of many branches of applied sciences, such as material science, fundamental physics, fluid dynamics, physiology and space medicine, plant and cellular biology, combustion physics. All require conducting experiments in weightlessness (Seibert et al. 2001; Beysens and van Loon 2015; Pletser 2018). Additionally, the current trend of space commercialization (de Crombrugghe and Pletser 2017) induces a growing interest for weightlessness facilities. Yet, the number of solutions able to reproduce microgravity on Earth is limited and they all have their limitations (Herranz

Camille Gontier

camille@lide.space

1 LIDE Space, Louvain-la-Neuve, Belgium

2 Institutes of Mechanics, Materials and Civil Engineering, Université catholique de Louvain, 1348 Louvain-la-Neuve, Belgium

3 Department of Physiology, University of Bern, Bühlplatz 5, 3012 Bern, Switzerland

4 Blue Abyss, Liverpool, United Kingdom et al. 2013; de Crombrugghe and Pletser 2017). Sounding rockets, aircraft parabolic flights and drop towers are today's most valued microgravity platforms. However, their cost and lead times make them unaffordable or unpractical for many space actors, such as small start-ups, universities, or student projects (Council 2011). In particular, parabolic flights performed with aircraft were introduced in the 50's, namely to study the effect of reduced-gravity environments on the human body (Haber and Haber 1950). They then developed as an easy-to-access and muti-purpose microgravity platform, which however has become more and more expensive and which nowadays also suffers from long lead times.

There have been some recent interests in alternative, less costly solutions for microgravity testing. For instance, parabolic flights can be performed with light single-engine piston aircraft (de Crombrugghe and Pletser 2017), as was demonstrated by flight test campaigns operated with a Cessna 206 (Selig et al. 2016, 2017, 2018, 2019) and a CAP 10 (Perez-Poch et al. 2016a; Brigos et al. 2014). Microgravity was achieved for approximately 8 seconds during each parabola, with great operational flexibility.

Sailplanes are also potentially favourable platforms for reaching weightlessness. A first quantification of gliders 
0-g flight capabilities has been realized (Pletser et al. 2017a, b) using a Grob G-103 Twin II. The results showed that:

- Weightlessness can be achieved continuously for up to 6 seconds per parabola;

- A 20 to 25-minute flight allows performing up to 21 parabolas;

- The estimated order of magnitude of the average parasitic acceleration in weightlessness ranges between $10^{-2}$ and $10^{-1} \mathrm{~g}$.

The goal of the present study is to refine these results and to provide a more complete and systematic quantification of gliders 0 -g flight capabilities. We present the results of a flight test campaign of parabolic flights realized with an Alexander Schleicher ASK-21 (which has similar characteristics to the G-103). Data were collected using custom-made recorders and accelerometers which allow us to precisely identify the level of $0-\mathrm{g}$ achieved during parabolas, and their duration.

In this article, we first expose in Section Context and Motivations the context of research and tests in microgravity, and we elaborate on the potential benefits of the development of low-cost sailplane-based microgravity platforms. In Section Description of the Experiments and Collection of Data, we introduce the flight test campaign that aimed to better quantify the capabilities of sailplane-operated parabolic flights. We describe the sensors that are used to collect data, and the additional systems involved. Section Systematic Quantification of Gliders 0-g Flight Capabilities is dedicated to an analysis of the flight test results, with a specific focus on the microgravity phase. A comparison between the vibration levels measured in a glider and in a light motorized airplane is also presented. Finally, in Section Discussions, we discuss some operational considerations specific to glider flights, and we put in perspective the associated advantages and limitations with existing solutions.

\section{Context and Motivations}

\section{Applications of 0-g Environments on Earth}

The range of scientific fields that are related to reducedweight environments is extremely large. Their thorough enumeration would be quite tedious. We refer to the reviews by Seibert et al. (2001) and Pletser and Harrod (2014) and Pletser (2016b) for a mere overview. Additionally, we here also provide three major examples of experiments that illustrate how access to weightlessness is fundamental to modern science.

Fluid dynamics are notoriously difficult to model in weightlessness, which is a major impediment to spacecraft control efficiency. Indeed, sloshing in tanks of liquid propellants rockets or satellites leads to disturbing torques that are hardly predictable and controllable. The FLUIDICS (Fluid Dynamics in Space) experiment, run on board the International Space Station (ISS), is an attempt to better understand liquid sloshing and wave turbulence phenomena (Dalmon et al. 2019).

The equivalence principle can be measured by comparing the accelerations of two atoms having different masses and structures using a two-species atomic interferometer. On Earth, measured atoms are subject to gravity and ultimately fall off the recording area, while weightlessness allows for longer, and thus more precise, recordings. The ICE (Coherent Source Interferometry for space) is an experiment that flew several times on European Space Agency (ESA) ${ }^{123}$ and French Centre National d'Études Spatiales (CNES) parabolic flight campaigns to prepare for an ISS experiment (Stern et al. 2009; Geiger et al. 2011; Ménoret et al. 2011; Barrett et al. 2016).

The Material Science in Variable Gravity Group of the University of Bremen (Julia Tielke 2018; Thode et al. 2018) is especially interested in seeing how reduced or high gravity levels impact material synthesis and properties. To this end, they developed the Self-Propagating High-Temperature Combustion Synthesis Capsule (SHS) meant to be tested within the ZARM drop tower. It is equipped with two monitored reaction chambers, and allows to study how material structures are affected by weightlessness.

Besides fundamental research, weightlessness is also used to test future equipment for the ISS and other space systems. For instance, heat dissipation systems and water supply systems, of which the optimal operation envelope depends on applied accelerations, are tested during numerous parabolic flights campaigns before being installed.

With the additional and ever-growing interest in CubeSats, and in space exploration in Low Earth Orbit (LEO) and beyond in general, there is nowadays a clear rise in the demand for conducting experiments and testing components in microgravity. Finally, new fields are also looking into the potential benefits of microgravity such as $3 \mathrm{D}$ printing,

\footnotetext{
${ }^{1}$ Test of Weak Equivalence Principle with cold atoms. Experiment Record $\mathrm{N}^{\circ}$ 9290. ESA website http://eea.spaceflight.esa.int/portal/ exp/?id=9290, last accessed 21st March 2020.

${ }^{2}$ ICE: Atomic interferometry with cold atoms in microgravity. Experiment Record $N^{\circ}$ 9317, Erasmus Experiment Archive. ESA website http://eea.spaceflight.esa.int/portal/exp/?id=9317, last accessed $21 \mathrm{st}$ March 2020.

${ }^{3}$ Weak Equivalence Principle in Space. Experiment Record $N^{\circ} 9463$. Erasmus Experiment Archive. ESA website http://eea.spaceflight.esa. int/portal/exp/?id=9463, last accessed 21st March 2020.
} 
medicine, etc. Overall, this rise in demand comes mainly from smaller research organisations or privately funded commercial entities which are much more sensitive to the cost and lead time of access to traditional platforms.

\section{Existing Solutions for Ground-Based Microgravity Platforms and Their Limitations}

In spite of the uninterrupted growth of microgravity customers that we highlighted in the previous section, the current offer of weightlessness solutions has not really evolved over the last decades. Hence, today, access to microgravity research might prove too expensive and complicated for many space actors, and especially for newcomers in the space industry. The average cost and lead time for the most popular solutions are summarized by Pletser et al. (2017b) and further discussed in Section Discussions.

Only few laboratories and research organizations can afford parabolic or suborbital flights, not to mention the cost of sending an experiment to the ISS. Parabolic Flights are often used to conduct experiments and train astronauts in microgravity. For example, ESA and CNES conduct two campaigns per year, and the Deutsches Zentrum für Luft- und Raumfahrt e.V. (DLR, German Aerospace Center) conducts alternatively one or two campaigns a year, with an average of 12 to 15 experiments per campaign (summing up to 60 to 90 experiments per year on the ESA-CNESDLR Airbus A310) (Pletser et al. 2014, 2015, 2016b, c). Generally, parabolic flights are performed with large airliners, although smaller-size aircraft can also be used (see a review in Pletser et al. 2016b). Solutions based on those are currently emerging, and will contribute to expanding the microgravity offer (Perez-Poch et al. 2016a; Selig 2019).

For Sounding Rockets, REXUS is the leading initiative in Europe, conducting one campaign per year, only for students.

Among ground-based solutions, drop towers are used around the world (USA, Europe, China, and Japan) to achieve up to 9 seconds of free fall. The highest in Europe is the ZARM tower located in Bremen, Germany ${ }^{4}$. Although drop towers allow to reach a very good level of microgravity (with a residual acceleration during the drop typically as low as $10^{-6}$ to $10^{-5} \mathrm{~g}$ ), their main weaknesses include high $g$ loads during launch (up to $30 \mathrm{~g}$ for the ZARM catapult mode) and recovery, and long lead times (the ZARM drop tower is operated with only 12 drops per week $)^{5}$.

\footnotetext{
${ }^{4}$ The Bremen Drop Tower. Zentrum fur Angewandte Raumfahrttechnologie und Mikrogravitation (ZARM). Universitat Bremen. https:// www.zarm.uni-bremen.de/en/drop-tower/general-information.html, last accessed 21st March 2020.

${ }^{5}$ ZARM Drop Tower User Manual. https://www.zarm.uni-bremen. de/fileadmin/user_upload/drop_tower/Users_Manual_0412.pdf, last accessed 10th June 2020.
}

To a lesser extent, clinostats and random position machines can be used to reproduce microgravity from a statistical perspective, but these usually only accommodate small samples and simulate omnidirectional gravity rather than microgravity (Brown et al. 1976), and are thus mostly used to study cellular biology and plant growth (Herranz et al. 2013; Hasenstein et al. 2015). In Europe, all these platforms are being currently used by laboratories and by the industry.

Students can also access some of these facilities through ESA-financed program (Fly your thesis, Drop your thesis, Spin your thesis) (Callens et al. 2011). But these programs are limited in scope and capacity and the lead times to get access to one of the test slots are relatively long. While these initiatives are very beneficial for microgravity research in Europe, they also epitomize the demand for more accessible test opportunities in microgravity conditions.

\section{Motivations for Sailplane-Operated Parabolic Flights}

Sailplanes are commonly operated in many places of the world. Moreover, a majority of the training glider currently in use are suitable for parabolic flights, as the structural loads endured during the parabola are compatible with most of today's glider flight envelopes (see Section 4). Besides, the costs of operation of a glider are relatively small.

In order to guarantee the safety and the quality of the parabolic flight, one should however ensure that:

- pilots are properly trained for the manoeuvres in all phases of a parabolic flight;

- the subject of the experiment/test is correctly installed and fastened in the glider.

Provided that these two conditions are filled, sailplane parabolic flights could be used to endorse a large number of microgravity experiments for research, academic, and industrial purposes. This could further contribute to reducing the pressure on well-established microgravity providers, allowing customers to realize preliminary studies and tests on (parts of) their experimental devices, at reduced expenses.

\section{Description of the Experiments and Collection Of Data}

Data presented hereafter were collected during the first phase of a flight test campaign which consisted of 7 flights, totalling 74 parabolas, conducted between September 2018 and September 2019. All flights were performed in smooth air conditions in order to minimize the perturbations due to atmospheric turbulence. To ensure smooth flights, we 
systematically checked for a stable stratified atmosphere, no visible convective clouds, a low solar radiation (morning or late afternoon flights), and a surface wind intensity lower than $10 \mathrm{~m} / \mathrm{s}$. Recordings were obtained on board an ASK21 two-seat glider (see Fig. 1). The manufacturer specifies a $\mathrm{g}$-limit of $+6.5 \mathrm{~g}$ and $-4.0 \mathrm{~g}$ for structural reasons. This flight envelope largely covers the needs of 0 -g parabolic flights, and is similar to the limitations of other two-seat plasticmade training gliders (e.g. DG-505, DG-1000, G-103).

\section{Data Collection: sensors and Means of Measurement}

During the flight tests, we used three independent devices in order to record data for the quantification of the parabolas. The first Flight Data Recorder (FDR-1) is based on an Arduino-YUN rev2 board, equipped with a microcontroller for data acquisition and processing, and a Linux microprocessor which was used here for real-time data display (see Section Real Time Display). The FDR1 was fitted with a DP0107 Global Navigation Satellite System (GNSS) module for geo-localization and with a MPU-9250 Inertial Measurement Unit (IMU) providing measurements of the acceleration, the rotation rate and the magnetic field intensity in 3-D. In particular, acceleration data can be collected at a maximum rate of $4 \mathrm{kHz}$, with a corresponding bandwidth of $1.13 \mathrm{kHz}$. However, for the present application, we initially aimed for a synchronized data acquisition at a rate of $10 \mathrm{~Hz}$, which is the largest allowed by the GNSS module. In practice, because of the high workload demanded from the processing of both sensors data, and also accounting for the communication between the microcontroller and the Linux processor, the actual sampling rate varies between 3 and $5 \mathrm{~Hz}$. In order to reduce as far as possible the aliasing of the IMU measurements, the sensor-embedded digital low-pass filter is set to restrict the bandwidth to $5 \mathrm{~Hz}$.

The second data recorder uses a Raspberry $\mathrm{Pi} 3$ microcomputer, wired with a smart Bosch BNO055 absolute orientation sensor. The latter processes data from

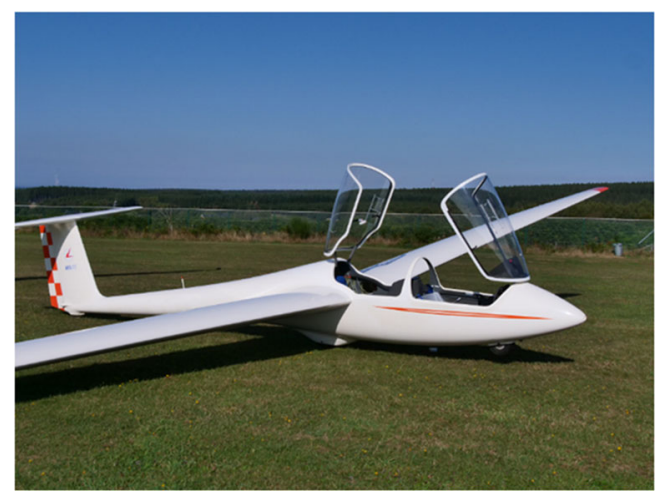

Fig. 1 The ASK-21 used for the flight test campaign an internal Inertial Measurement Unit (IMU) equipped with an accelerometer, a gyroscope and a magnetometer. Generally, obtaining the actual aircraft absolute orientation from the related measurements is difficult, even more so on low cost real-time systems (Lie and Gebre-Egziabher 2013). The BNO055 chip is programmed to processes all the sensors data and to output readily usable information in the form of quaternions, Euler angles or vectors. This abstraction level removed our concerns about sensor fusion and real-time requirements. The algorithm developed by the authors could hence focus on combining the orientation and acceleration data to get meaningful insights for the pilots during post-flight reviews. Overall, FDR-2 has a sampling and writing frequency of about $25 \mathrm{~Hz}$.

Finally, the built-in accelerometer sensor of a smartphone was used as a third (back-up) sensor. Advantageously, the sampling frequency could be increased to $50 \mathrm{~Hz}$.

All sensors were installed in the backseat of the glider, as shown in Fig. 2, in order to minimize their distance to the centre of gravity (CG) of the sailplane. Tape was used as the easiest and most efficient solution to attach the recorders. Inevitably, the offset distance between the sensor and the CG introduces a spurious acceleration in the recordings during changes in attitude of the aircraft, as noticed by Karmali and Shelhamer (2008). We here verify the negligible character of this effect by estimating the magnitude of the parasitic acceleration. During the parabola, the glider pitch rate is of the order of $0.26 \mathrm{rad} / \mathrm{s}$ (as it goes from $\mathrm{a}+45^{\circ}$ to $\mathrm{a}-45^{\circ}$ pitch angle in approximately $6 \mathrm{~s})$.

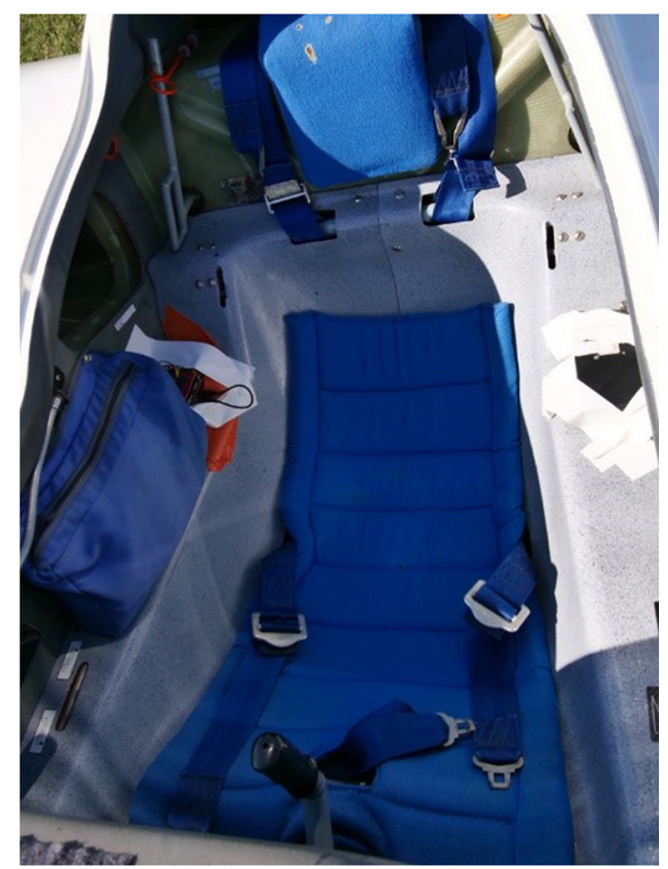

Fig. 2 Mounting of the sensors in the backseat of the glider (FDR-1 on the right, FDR-2 on the left) 
As the sensor is located roughly $0.5 \mathrm{~m}$ in front of the $\mathrm{CG}$, the centripetal acceleration is of the order of $3.410^{-3} \mathrm{~g}$. This is indeed small with respect to the mean residual acceleration experienced during the parabolas (as detailed hereafter).

\section{Real Time Display}

In 2018, a first series of exploratory flights were conducted. From this early experience, it became clear that specific pilot training was required to guarantee the good quality of the parabolas. This was also pointed out by Brigos et al. (2014). Meanwhile, our pilots also identified the need for a real-time feedback of the g-force experienced by the payload in order to fine-tune the sailplane trajectory. In fact, aviation accelerometers exist but are not part of the mandatory instruments for gliders (there were none in the glider which was used for the present study). Moreover, the sensor should be located as close as possible to the experiment (likely in the backseat) for a higher precision.

In order to allow for real-time acceleration display, the FDR-1 hosts a Hypertext Transfer Protocol (HTTP) server embedded with a Javascript runtime environment. The server is provided with the real-time measurements and is programmed to pack them inside a graphical user interface and return them on HTTP requests. We use the Arduino's capability of creating a Wi-Fi hotspot for handling wireless connections between the FDR and external devices. As a result, a smartphone with a standard internet browser can be used as a monitor for our digital accelerometer. Figure 3 shows an example of the view displayed to the pilot.

A satisfactory latency of less than $0.25 \mathrm{~s}$ is observed between the time of measurement and the time of display, and the refresh rate of the display is close to the sampling rate of FDR-1. This shows that the entire data processing

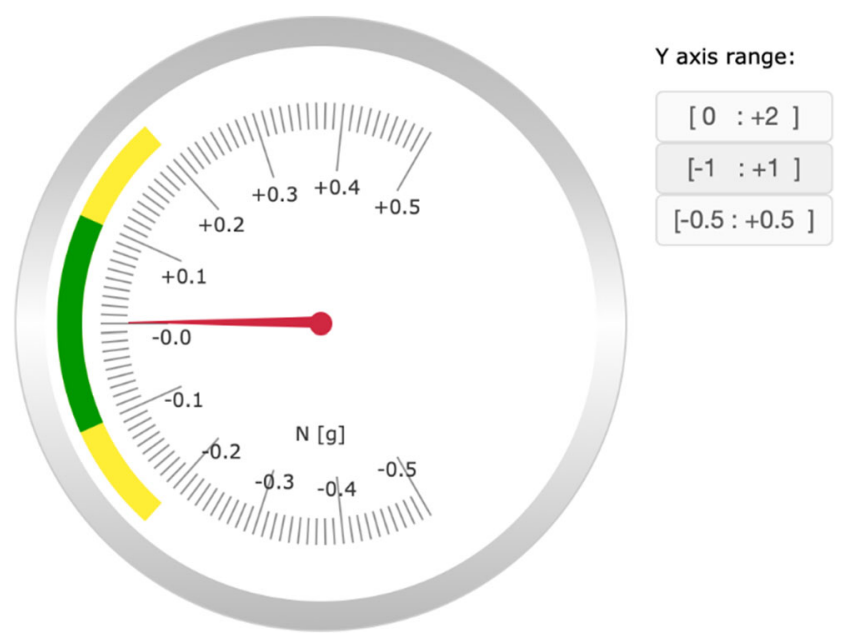

Fig. 3 Example view of the pilot display generated by FDR-1, showing the current load factor and communication chain only introduces a moderate lag. However, feedback from the pilots indicates that an even higher refresh rate would allow them to refine the parabolic trajectory and smoothen their actions on the glider flight controls.

\section{Systematic Quantification of Gliders 0-g Flight Capabilities}

\section{Parabola from an Instantaneous Perspective}

Unpowered aircraft can hardly reach the same quality of weightlessness as conventional aircraft used for parabolic flights, nor will they be able to sustain parabolas nearly as long. Indeed, at first order, the duration of the parabola is proportional to the injection velocity (i.e. the velocity at which the parabolic trajectory is initiated), which gives a clear advantage to fast flying airplanes for maximizing the duration of the free fall. Nevertheless, some aspects of the $0-\mathrm{g}$ flight capabilities of a sailplane are here further investigated. The precision of the trajectory is quantified, also attesting the reproducibility of microgravity conditions. The influence of the aerodynamic drag and the environmental noise are also assessed.

In this article, all accelerations are given in the body axis frame, with the $x$-axis pointing forward out of the nose (i.e. in the axial direction), the $\mathrm{z}$-axis pointing downward (i.e. in the vertical direction) and the $y$-axis to form an orthonormal frame, as illustrated in Fig. 4. More precisely, when the glider is in a steady straight flight (at the best glide speed), the z-axis points in the direction of the gravity. We tolerate an error of alignment of up to $2^{\circ}$ (due to sensor positioning).

In practice, the flight procedure consists in performing several parabolas in sequence, starting from an initial dive used to build up speed and reach the initial velocity. Figure 5 shows the time history of the vertical acceleration divided by the gravitational acceleration $g$ (i.e., the load factor) and the altitude during a sequence of parabolas.

In order to maximize the duration of the parabolas, the injection velocity should be as high as possible. However, in addition to the never-exceed velocity $(\mathrm{VNE}=250 \mathrm{~km} / \mathrm{h}$ for the ASK-21), an even more restrictive constraint stems from the flight envelope.

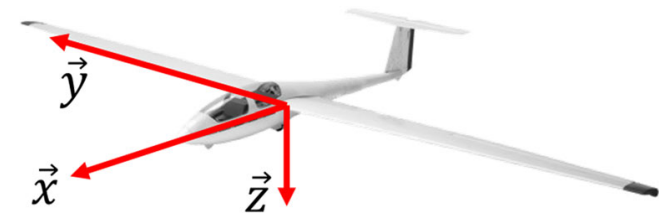

Fig. 4 Body frame axes 

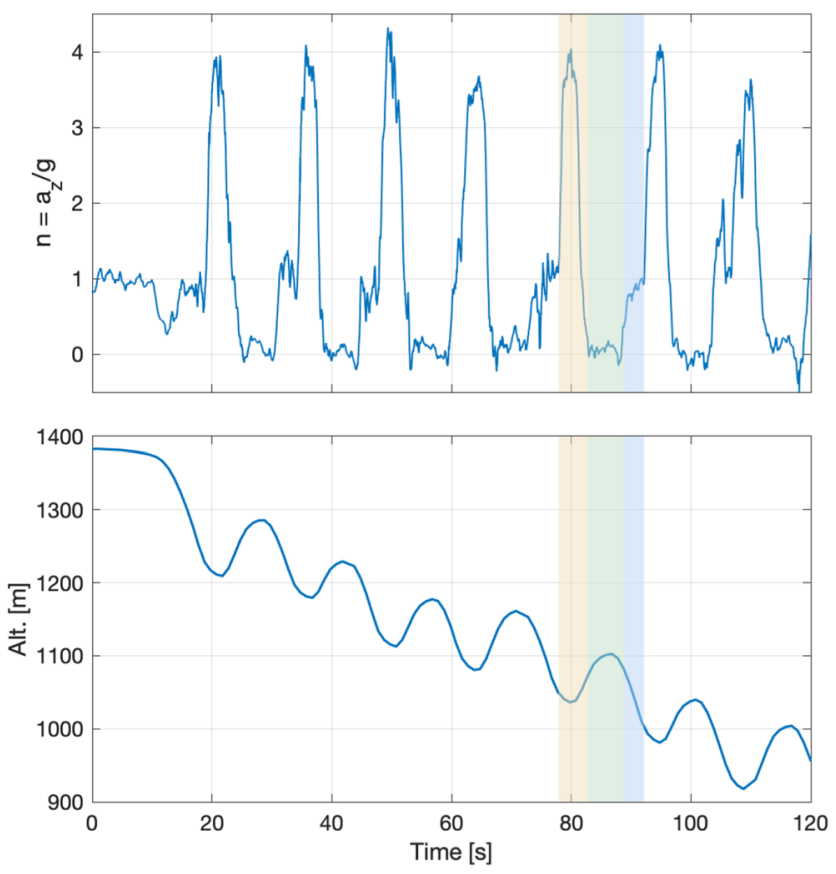

Fig. 5 Time history of the vertical acceleration (load factor, top) and the altitude (bottom) during a portion of flight number 3 , with the initial dive and 7 subsequent parabolas. For the fifth parabola, shaded areas identify the pull-up phase (yellow), the parabolic phase (green) and the acceleration phase (blue)

One must indeed verify that the initial velocity will not lead to load factors exceeding the threshold during the pullup between two parabolas. In this case, we intentionally set this limit to $4 \mathrm{~g}$ in order to alleviate the loads on the glider. Therefore, we select a target initial velocity of $200 \mathrm{~km} / \mathrm{h}$ which gives an injection velocity of $150 \mathrm{~km} / \mathrm{h}$.

The sequence of parabola is made of several periods, and it can be clearly seen from the acceleration profile that each period can be decomposed in three phases. Starting when the initial velocity is reached, the first phase is the pull-up where the glider transitions from a dive to a pitch angle of $+45^{\circ}$ (yellow shaded area). The second phase starts at the injection: the pilot promptly pushes the stick forward until the $0 \mathrm{~g}$ is reached, and by doing so he initiates the parabola. This phase lasts for about 6s (green shaded area) and will be further analysed here after. In the third phase which lasts for about $3 \mathrm{~s}$, the glider is maintained in a nose down attitude in order to recover the initial velocity required for the next period in the sequence (blue shaded area).

\section{Parabola from a Statistical Perspective}

In order to better characterize the $0-\mathrm{g}$ phase which is here arbitrarily defined as the portion of the parabola with $\frac{a_{z}}{g}<0.2$, the accelerations measured by the back-up high frequency $(50 \mathrm{~Hz})$ sensor were used to derive statistics over a set of 30 parabolas. The acceleration profile of every flight was first synchronized based on the time at which the threshold of $\frac{a_{z}}{g}<0.2$ was reached, and then the average and the standard deviation were computed from the aligned data. The results are shown in Fig. 6. We stress that the convergence of the statistics is partially limited due to the relatively small number of parabolas involved. However, the ensemble size should be sufficient to identify the main trends.

The average vertical acceleration shows that the pilot is indeed able to maintain the glider in a fairly constant reduced gravity environment. The microgravity phase is $5.5 \mathrm{~s}$ long on average, with a standard deviation of about $1 \mathrm{~s}$. Because it is quite short as compared to conventional parabolic flights, it is hard to further decompose the microgravity phase as was proposed in Lambot and Ord (2016).

Importantly, the standard deviation of the vertical acceleration is lower than $0.1 \mathrm{~g}$. This mainly characterizes the repeatability of the manoeuvre and the level of smoothness acquired in piloting the trajectory of the glider. Those two characteristics are mostly dependent on the pilot and on his experience. Notice that the cause of the standard deviation is not to be found in measurement noise or vibrations, as will be shown in Section Jitter Analysis and Comparison with Aircraft Capabilities.

The average vertical acceleration during the parabola is almost zero. The small residual offset, together with the standard deviation itself, could be both further decreased with an improved real-time display system, and will be also reduced with the increasing experience and practice of the pilots. It is worth mentioning that the targeted mean level of weightlessness can also be adapted to simulate Moon $(0.165 g)$ or Mars $(0.374 g)$ surface gravities, just as in traditional parabolic flights (de Crombrugghe and Pletser 2017).

Strictly speaking, because the glider has no engine, perfect zero-gravity conditions cannot be reached as opposed to propelled aircraft, for which the residual drag force can be compensated by the engine thrust. The parasitic drag experienced by the glider on the parabolic trajectory results in an axial acceleration which cannot be zero. It is here measured to be smaller than $0.02 \mathrm{~g}$ on average (see Fig. 6), which is compatible with a rough estimation based on the glider technical specifications.

Indeed, we assume that the parasitic drag is $50 \%$ of the total drag at the best glide angle $(\gamma)$. This stems from a simple and commonly used aircraft polar model, $C_{D}=$ $C_{D_{0}}+k C_{L}^{2}$, where $C_{D}$ is the total drag coefficient, $C_{D_{0}}$ is the parasitic drag coefficient and $k C_{L}^{2}$ is the induced 

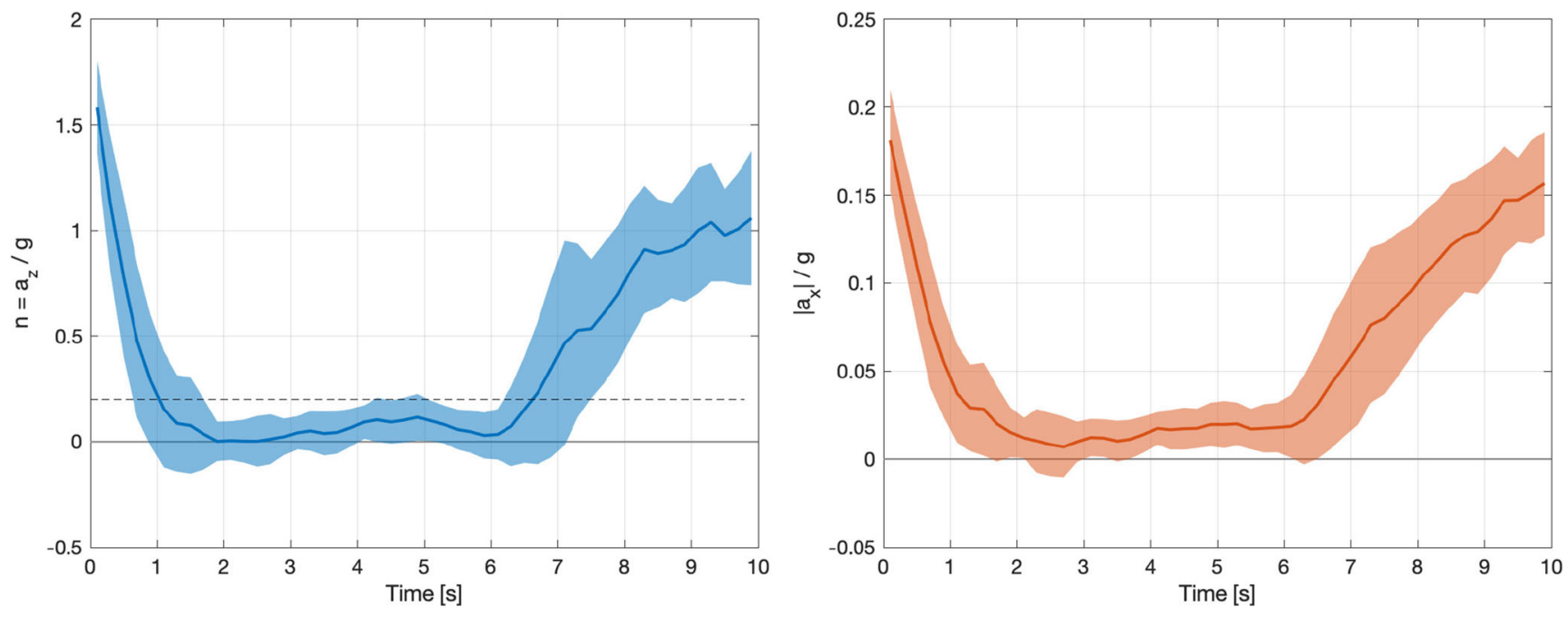

Fig. 6 Average vertical (left) and axial (right) accelerations (resp. $a_{z}, a_{x}$ ) measured during a parabola, and their standard deviations (shaded areas)

drag coefficient. Based on the technical specifications of the glider $^{6}$, we then find a drag coefficient of

$C_{D_{0}}=\frac{1}{2} \frac{1}{\gamma} \frac{M g}{\frac{1}{2} \rho V_{\gamma}^{2} S}=0.012$

with $S$ the reference surface, $V_{\gamma}$ the best glide angle velocity and $\rho$ the air density. With a glider mass $M=550 \mathrm{~kg}$, this should correspond to an acceleration of the order of $0.025 \mathrm{~g}$. This axial acceleration is however small compared to the variations in vertical acceleration shown above.

\section{Jitter Analysis and Comparison with Aircraft Capabilities}

In addition to a low average acceleration, a good-quality microgravity environment is also characterized by reduced high-frequency vibrations, an equally important criteria as underlined in Carr et al. (2020). Gliders do not suffer from the increased level of vibrations generated by piston engines, which had previously been identified as one of the main drawbacks of using light aircraft for weightlessness studies (Selig et al. 2016; de Crombrugghe and Pletser 2017). In order to verify this assertion, we compare the acceleration measured during a standard glider flights with new data collected in a General Aviation (GA) aircraft. The latter recordings were obtained on board a Diamond DA40TDI, a four-seat single-engine piston light aircraft equipped with a $135 \mathrm{Hp}$ Thierlet Centurion Diesel-injected engine.

As we are interested in the vibration level in each aircraft type, we compare the norm of the acceleration vector collected in similar flight conditions. Both data sets are obtained from our back-up sensor with the highest sample

\footnotetext{
${ }^{6}$ See the aircraft flight manual provided by the manufacturer at https:// www.alexander-schleicher.de/en/flugzeuge/ask-21-b/
}

frequency available to us $(50 \mathrm{~Hz})$. The data post-processing is similar to what is proposed in Carr et al. (2018), and involves the following steps on the norm of the measured acceleration vector:

- Data segmentation. We manually select two flight segments of approximately 8 minutes. For each aircraft, the selected sequence corresponds to similar cruise conditions, including straight flight and turns.

- Filtering. A high-pass filter is applied on data to remove the low frequency component from the jitter analysis (see Fig. 7). A fifth order digital Butterworth filter with a cutoff frequency of $1 \mathrm{~Hz}$ was designed using the Scipy Signal Processing toolbox in Python (Jones et al. 2001) and applied to data forwards and backwards. The attenuation of the filter is presented in Fig. 8, for frequencies close to the cutoff. We clearly see that, above $1.5 \mathrm{~Hz}$, data are unaffected and we retain the meaningful information for the present analysis.

- Sigma computation. The variable of interest to be compared among recordings is the standard deviation of the filtered acceleration.

- Power Spectral Density (PSD) computation. For further analysis, the PSD of the recordings is computed using Welch's average periodogram method, see Fig. 9. The size of the Fast Fourier Transform window here used is 512.

Eventually, we compute that the standard deviation of the filtered acceleration is $0.0357 \mathrm{~g}$ for the glider, and $0.0421 \mathrm{~g}$ for the GA aircraft, which hints at a slight advantage of sailplanes. This is confirmed by the PSDs (Fig. 9) from which one notes the higher energy in the filtered acceleration signal of the GA aircraft. Clearly at frequencies higher than $5 \mathrm{~Hz}$, there is a difference of up to $10 \mathrm{~dB} / \mathrm{Hz}$ in favor of the glider. 

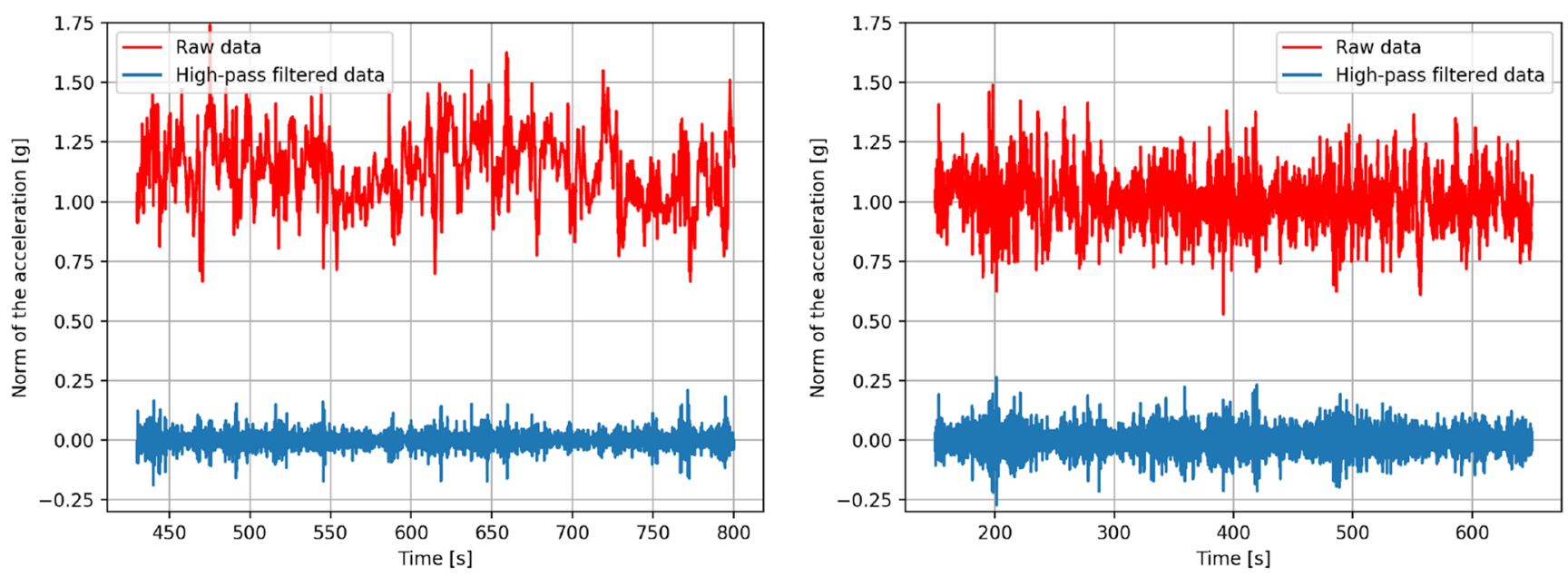

Fig. 7 Measured and filtered accelerations for glider (left) and GA aircraft (right)

Importantly, the present frequency analysis only enables the comparison of spectra up to $50 / 2=25 \mathrm{~Hz}$, i.e. the Nyquist frequency for our recordings. However, this frequency is smaller than the nominal engine regime, which is typically above 2000 revolutions per minute (i.e. approximately $33 \mathrm{~Hz}$ ). Consequently, we are not able to assess the effect of the engine frequency and harmonics which should cause peaks on the PSD. The energy stored in these peak frequencies can here not be quantified. Adding to the already lower broadband noise measured below $25 \mathrm{~Hz}$ in the glider, the engine-related peak vibrations can only be detrimental to the experimental conditions, hence supporting our conclusion in favor of sailplanes.

In order to systematically compare the level of jitter for sailplanes and engine aircraft, and their capacities in terms of achieved weightlessness, we should not only have used data collected in cruise conditions, but also data recorded during parabolas. Obviously, the absence of vibrations during that phases of the flight is critical. For the present flight test campaign however, we were not authorized to perform parabolas with the Da-40. Indeed, there are several risks associated with sustained weightlessness in a single engine piston aircraft. Often, the lubrication system of GA engines relies on gravity for the proper feeding of oil to the lubrication pump. Prolonged microgravity could thus lead

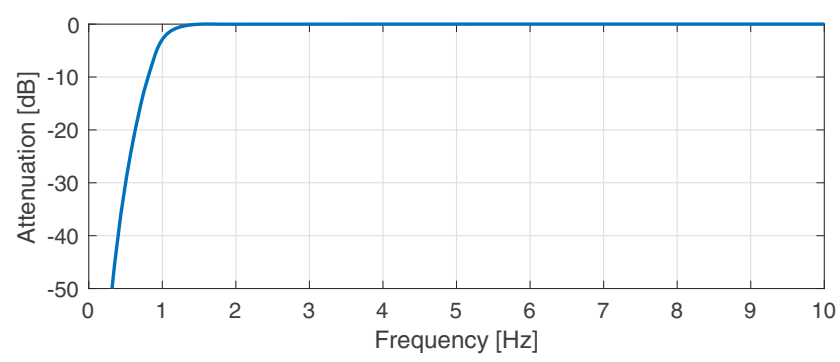

Fig. 8 Attenuation of the $5^{\text {th }}$ order Butterworth high-pass filter to oil starvation of the engine, causing the risk of engine damage due to the absence of lubrication. Depending on the engine design, this remark could also apply to the fuel system.

\section{Discussions}

\section{Potential volume of flights}

In this section, we determine the average number of parabolas achievable per flight, and we discuss the yearly availability rate of glider-operated parabolic flights.

Over one flight, the potential number of parabolas obviously depends on the release altitude of the glider. Using the characteristics of every flight in our flight test campaign, we determine that the average altitude loss per parabola is $50 \mathrm{~m}$. This is confirmed by the altitude profile obtained from GNSS data.

Operationally speaking, the glider is preferably towed up to a height of about $1000 \mathrm{~m}$. Accounting for a reserve height of $300 \mathrm{~m}$ necessary for manoeuvring and for the execution of the standard landing pattern, the remaining height of $700 \mathrm{~m}$ can be used for parabolic flight. Therefore, one can expect to perform 14 to 15 parabolas per flight. Furthermore, several flights can be performed in a row to increase the number of parabolas performed in a day, as the duration of one of these typical flights is around 20 minutes.

Over a year, the cumulative availability is difficult to estimate, mainly because the operation of sailplanes is dependent on weather conditions, as they are bound to fly under visual flight rules (similarly to general aviation aircraft, and as opposed to standard commercial aircraft). Rain, strong winds, low ceiling or reduced visibility typically prevent safe operation. Furthermore, typical weather conditions depend on the location. For 

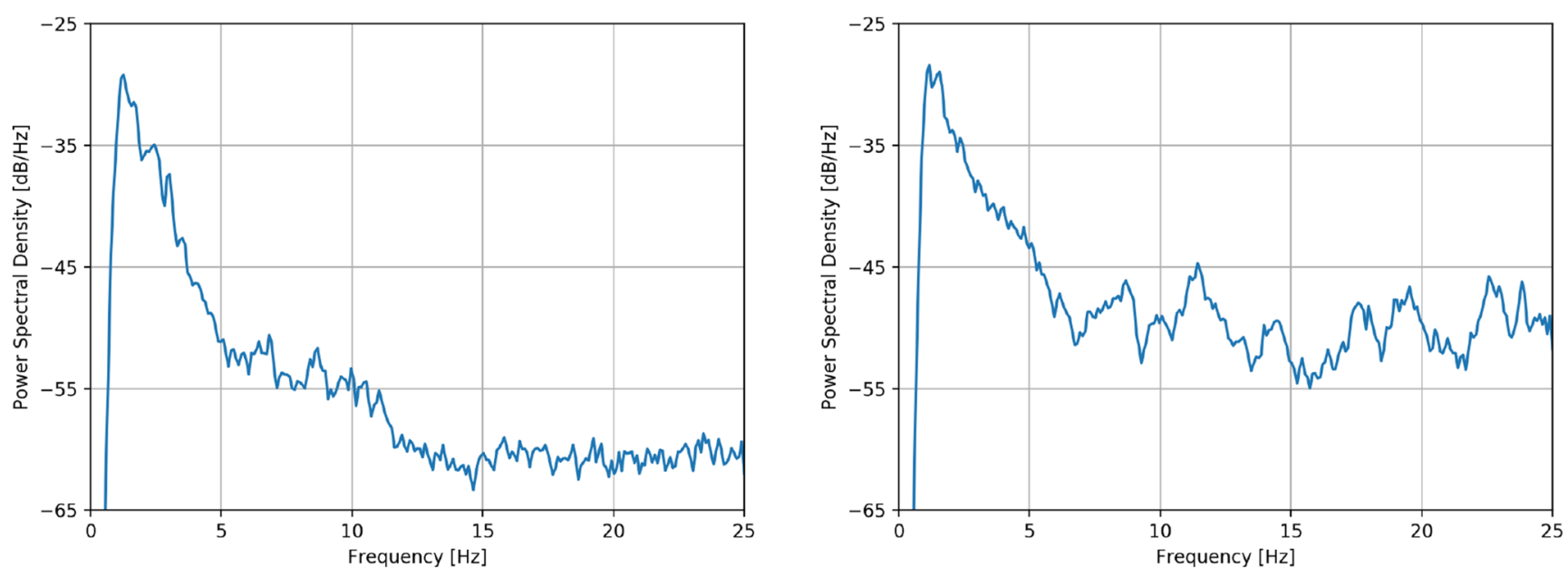

Fig. 9 Power spectral density of the filtered acceleration for glider (left) and GA aircraft (right)

example, in southern Europe, gliders are often constantly operated all year long, while it is not uncommon to observe a drop in activity during winter time in regions up north.

Nevertheless, we propose a provisional estimation of the total number of flights per year. Assuming that the conditions required for flying are met once in three days on average (which is quite conservative), we postulate that a single airframe can perform approximately 100 flights per year (or equivalently, 1500 parabolas per year). Notice that this number is compatible with (i.e. smaller than) the typical number of flights performed by a training glider in the average European gliding club.

\section{Payload Size and Weight Limitations}

The backseat of the glider could accommodate a large range of experiments, provided they respect the limitations of mass and volume. For instance, the ASK-21 manufacturer specifies that the mass in the back seat must not exceed $110 \mathrm{~kg}$, thus limiting the experimental payload mass. This number can be used as a rule of thumb for other types of sailplanes. The room available depend on the glider type, but the usable volume roughly fits a cube with $50 \mathrm{~cm}$ edges.

Payload limitations, in terms of weight, are thus of the same order of magnitude as for the GA-aircraft based platform proposed by Selig et al. (2016, 2017, 2018, 2019) (i.e. $150 \mathrm{~kg}$ ), and are a bit more restrictive in volume (i.e. $1000 \mathrm{~L})$.

\section{Comparison with Existing Solutions}

In order to enable the comparison between glider parabolic flights and other existing solutions, we provide an estimation of several metrics in Table 1.

Data are adapted from Pletser et al. (2017b). The estimated cost per experiment assumes:
- 1 experiment per fall in drop towers, 1 per flight for gliders, 12 per flight for engine-based aircraft, and 5 per sounding rocket;

- Prices do not include depreciation and cost of development;

- Cost for experiments in the ISS includes a $20 \mathrm{k} € / \mathrm{kg}$ cost for the launch itself.

The main advantages of the sailplane-operated parabolic flights include a low cost per experiment, the geographical proximity between the provider of the experiment and the nearest airfield, and a shorter expected waiting time. The latter accounts for the above estimated number of flights per year, plus the fact that gliders could be operated in different locations worldwide. Notice that these advantages are shared with solutions based on small motorized aircraft.

On the other hand, the principal limitations of gliders as microgravity platforms are the shorter consecutive duration in weightlessness and the larger residual/parasitic acceleration. Compared to engine-based aircraft, higher load factors are attained between parabolas (up to $4 g$, see Fig. 5), but these remain small or similar to the maximum accelerations experienced in other solutions. In spite of the narrow volume available in a glider, a human operator can be present on-board (as it is often the case for experiments in large aircraft), depending on the actual volume of the experiment. An experiment must either be relatively small to be operated by a human experimenter sitting in the backseat (as an operator or as the experiment subject), or be completely autonomous following a pre-programmed sequence, or feature a connection with operators on the ground via an up-/down-link capability. Besides, the limited volume in the cockpit contributes to enhancing the human operator safety by restricting body movement, unlike in large aircraft where there are substantial hazards due to potential unusual body attitudes. 
Table 1 Compared metrics for various microgravity platforms.

\begin{tabular}{|c|c|c|c|c|c|c|c|}
\hline Platform & $\mu g(g)$ & Duration & Max load $(g)$ & Volume $\left(\mathrm{m}^{3}\right)$ & Interaction & Waiting time & Cost/exp $(€)$ \\
\hline Drop towers & $10^{-3 \ldots-6}$ & $<5 \mathrm{~s}$ & 50 & $<1$ & $\mathrm{TC}$ & $\approx$ months & $\approx 10 \mathrm{k}$ \\
\hline Airliner parabolic flights & $10^{-2 \ldots-3}$ & $\mathrm{Csc} \approx 20 \mathrm{~s} \mathrm{Tot} \approx 600 \mathrm{~s}$ & 2 & $>10$ & Hum & $\approx$ months & $\approx 125 \mathrm{k}$ \\
\hline Small motorized aircraft & $10^{-1 \ldots-3}$ & $\mathrm{Csc} \approx 8 \mathrm{~s}$ Tot $\approx 150 \mathrm{~s}$ & $\approx 3$ & $\approx 1$ & TC/Hum & $\approx$ days & $\approx 1 \mathrm{k}$ \\
\hline Sounding rockets & $10^{-4 \ldots-5}$ & $5 . .13 \mathrm{~min}$ & $>5$ & $<1$ & $\mathrm{TC}$ & $>2$ years & $>400 \mathrm{k}$ \\
\hline ISS & $10^{-2 \ldots-5}$ & Years & $\approx 3$ & $>1$ & TC/Hum & $>5$ years & $1 \ldots .5 \mathrm{M}$ \\
\hline Glider parabolic flights & $10^{-1 \ldots-2}$ & $\mathrm{Csc} \approx 5.5 \mathrm{~s} \mathrm{Tot} \approx 80 \mathrm{~s}$ & 4 & $<1$ & TC/Hum & $\approx$ days & $<1 \mathrm{k}$ \\
\hline
\end{tabular}

Data adapted from Pletser et al. (2017b), Perez-Poch et al. (2016a), and Selig (2019). $\mu g=$ average residual acceleration, Csc $=$ consecutive, Tot $=$ total per flight, $\mathrm{TC}=$ Telecommand, Hum $=$ operator or human subject

Finally, the complexity of experiment preparation is strongly reduced with gliders and small aircraft as standard laboratory equipment could be most commonly used. This still requires integration and a minimum of safety check, but without endless administrative verifications, as certification of laboratory equipment is already available.

\section{Opportunity for Hyper-Gravity Testing}

It is to be noted that the authors of Pletser et al. (2017b) also assessed the possibility to use a sailplane to generate a high gravity environment (by flying high-bank steep turns) or quickly changing g-loads (by flying aerobatics manoeuvres like "chandelles"). Results show that gliders can achieve a g-load between 1.5 and $2 g$ for 20 to $40 \mathrm{~s}$, and to quickly vary the g-load between $0 g$ and $4 g$. These manoeuvres are not within the scope of the present study, but show that sailplanes may also be used to perform high-g experiments.

\section{Conclusion}

In this work, we showed that using gliders or general aviation aircraft to reach weightlessness would represent a step further in the evolution towards providing more accessible and flexible microgravity platforms. A microgravity platform based on sailplane gliders would be especially suitable for compact experiments and equipment that do not require a long-lasting microgravity test conditions and which do not require the very large payload capacities of conventional aircraft.

Typically, a single sailplane parabolic flight will provide approximately 15 test windows of up to $6 \mathrm{~s}$ duration each. Several flights can easily be performed in a row, allowing a cumulated total test time in quasi microgravity similar or longer than 0-g flights performed by wide-body aircraft. Furthermore, a single glider would be capable of supporting of the order of 100 flights per year (a conservative estimation). Hence, many experiments could greatly benefit from the use of a glider, provided that they can fit in the backseat volume (approximately $125 \mathrm{dm}^{3}$ ) and that they comply with the maximum $110 \mathrm{~kg}$ mass limit requirement, with or without a human investigator acting as operator or subject.

From the results of our flight test campaign, we measured that the excursion on the acceleration during the microgravity phase remains well below $0.1 \mathrm{~g}$. We showed that the achieved microgravity conditions are reproduced from one flight to another, and thus that the operation is well repeatable. We noted that the small variations, together with the absolute level of acceleration reached, are highly dependent on the pilot experience, and can thus be improved with training. Better controlled parabolas will be obtained provided that a visual feedback on the acceleration is provided to the pilot. The hardware solution that was presented here is currently being improved to eventually compensate for the potential absence of an approved gmeter in the cockpit. Further work will also consider using a reference-frame independent approach to detecting parabolas (Carr et al. 2018), which will allow mounting an accelerometer using a reference frame that is most relevant to any specific object/system under study.

Alternatively, general aviation aircraft can be used as microgravity platforms, offering even longer duration parabolas and convenient operational flexibility. Because of the piston engine, the level of vibration is higher in a light aircraft than in a glider, as confirmed by the measurements that were here presented and compared. Despite the augmented risk inherent to the operation of internal combustion engine in microgravity, small airplanes feature a larger experimental volume and a residual acceleration similar to those measured during glider parabolas. Along with gliders, they thus constitute cheap alternatives to conventional airliner parabolic flights.

Entering the 2020's, ISS operations may soon come to an end. New space stations that could be used by international partners include the future Chinese space station foreseen to be launch in 2022 and the Gateway, an international station to orbit the Moon in a few years. These new orbital platforms would yield the development 
of new systems and technologies which will likely require testing and verification in microgravity, prior to their installation. Following this potential increase in demand, there is an opportunity to augment the capacity for systematic 0 -g testing. The approach that we envision relies on the development of an offer for alternative (but complementary to existing solutions) low-cost platforms to conduct preliminary microgravity research and testing, under the form of glider and/or general aviation parabolic flights.

Acknowledgments The authors acknowledge the contribution of $\mathrm{N}$. Frischauf, P. Billuart and H. Delattre in collecting valuable flight test data, and the collaboration with Aeroclub Universitaire de Louvain through which the airframe was made available. They also express their gratitude to the personnel of UCLouvain-iMMC for technical advising and sharing of resources.

Funding Open access funding provided by University of Bern.

Author Contributions Test flights: Denis-Gabriel Caprace. Development of flight data recorders: Denis-Gabriel Caprace and Mohammad Iranmanesh. Formal analysis and investigation: Denis-Gabriel Caprace and Camille Gontier. All authors contributed to writing the article.

Data Availability Samples of data recordings are available in the following GitHub repository: https://github.com/camillegontier/ lide_data_samples.git. Post-processing routines are available upon reasonable request.

\section{Compliance with Ethical Standards}

Conflict of interests Denis-Gabriel Caprace, Camille Gontier, Mohammad Iranmanesh, Mehdi Scoubeau are involved in the establishment of a commercial company to provide glider-based microgravity services through LIDE.

Open Access This article is licensed under a Creative Commons Attribution 4.0 International License, which permits use, sharing, adaptation, distribution and reproduction in any medium or format, as long as you give appropriate credit to the original author(s) and the source, provide a link to the Creative Commons licence, and indicate if changes were made. The images or other third party material in this article are included in the article's Creative Commons licence, unless indicated otherwise in a credit line to the material. If material is not included in the article's Creative Commons licence and your intended use is not permitted by statutory regulation or exceeds the permitted use, you will need to obtain permission directly from the copyright holder. To view a copy of this licence, visit http:// creativecommonshorg/licenses/by/4.0/.

\section{References}

Barrett, B., Antoni-Micollier, L., Chichet, L., Battelier, B., Lévèque, T., Landragin, A., et al.: Dual matter-wave inertial sensors in weightlessness. Nat. Commun. 7, 1-9 (2016)

Beysens, D.A., van Loon, J.J.A. (eds.): Generation and Applications of Extra-Terrestrial Environments on Earth. River Publishers, Aalborg (2015). https://doi.org/10.13052/rp-9788793237544
Brigos, M., Perez-Poch, A., Alpiste, F., Torner, J., González Alonso, D.V.: Parabolic flights with single-engine aerobatic aircraft: Flight profile and a computer simulator for its optimization. Microgravity Sci. Technol. 26, 229-239 (2014). https://doi.org/10.1007/s12217014-9382-0

Brown, A.H., Dahl, A., Chapman, D.: Limitation on the use of the horizontal clinostat as a gravity compensator. Plant Physiol. 58, 127-130 (1976)

Callens, N., Ventura-Traveset, J., de Lophem, T.-L., de Echazarreta, C.L., Pletser, V., van Loon, J.J.: Esa parabolic flights, drop tower and centrifuge opportunities for university students. Microgravity Sci. Technol. 23, 181-189 (2011)

Carr, C.E., Bryan, N.C., Saboda, K.N., Bhattaru, S.A., Ruvkun, G., Zuber, M.T.: Acceleration profiles and processing methods for parabolic flight. NPJ Microgravity 4, 1-5 (2018)

Carr, C.E., Bryan, N.C., Saboda, K.N., Bhattaru, S.A., Ruvkun, G., Zuber, M.T.: Nanopore sequencing at mars, europa and microgravity conditions. bioRxiv https://doi.org/10.1101/2020.01.09. 899716 (2020)

Council, N.R.: Recapturing a Future for Space Exploration: Life and Physical Sciences Research for a New Era. The National Academies Press, Washington (2011). https://doi.org/10.17226/ 13048

Dalmon, A., Lepilliez, M., Tanguy, S., Alis, R., Popescu, E.R., Roumiguié, R., et al.: Comparison between the fluidics experiment and direct numerical simulations of fluid sloshing in spherical tanks under microgravity conditions. Microgravity Sci. Technol. 31, 123-138 (2019)

de Crombrugghe, G., Pletser, V.: Emerging microgravity platforms and their capabilities compared to the traditional offering. In: 68th International Astronautical Congress (IAC), International Astronautical Federation (IAF), Adelaide. (IAC-17-A2-5-10x39598) (2017)

Geiger, R., Ménoret, V., Stern, G., Zahzam, N., Cheinet, P., Battelier, B., et al.: Detecting inertial effects with airborne matter-wave interferometry. Nat. Commun. 2, 1-7 (2011)

Haber, F., Haber, H.: Possible methods of producing the gravity-free state for medical research. J. Aviat. Med. 21, 395-400 (1950)

Hasenstein, K.H., van Loon, J., Beysens, D.: Clinostats and Other Rotating Systems-Design, Function, and Limitations. In: Generation and Applications of Extra-Terrestrial Environments on Earth, vol. 14, pp. 147-156. River Publishers, Aalborg (2015)

Herranz, R., Anken, R., Boonstra, J., Braun, M., Christianen, P.C., de Geest, M., et al.: Ground-based facilities for simulation of microgravity: organism-specific recommendations for their use, and recommended terminology. Astrobiology 13, 1-17 (2013)

Jones, E., Oliphant, T., Peterson, P.: Scipy: Open source scientific tools for python (2001)

Julia Tielke, M.C.: One-step method to synthesize tungsten nanofluids in variable gravity. In: 69th International Astronautical Congress (IAC). International Astronautical Federation (IAF), Bremen. (IAC-18-C2-8-11-x46045) (2018)

Karmali, F., Shelhamer, M.: The dynamics of parabolic flight: Flight characteristics and passenger percepts. Acta Astronaut. 63, 594602 (2008). https://doi.org/10.1016/j.actaastro.2008.04.009

Lambot, T., Ord, S.F.: Analysis of the quality of parabolic flight. In: Next-Generation Suborbital Researchers Conference. ARC-EDAA-TN32699-1 (2016)

Lie, F.A.P., Gebre-Egziabher, D.: Synthetic air data system. J. Aircr. 50, 1234-1249 (2013)

Ménoret, V., Geiger, R., Stern, G., Zahzam, N., Battelier, B., Bresson, A., et al.: Dual-wavelength laser source for onboard atom interferometry. Opt. Lett. 36, 4128-4130 (2011)

Pletser, V., Harrod, J.: The science of gravity a new era of ESA experiments on parabolic flights. ESA Bulletin, European Space Agency, 22-30 (2014) 
Pletser, V., Rouquette, S., Friedrich, U., Clervoy, J.-F., Gharib, T., Gai, F., et al.: European parabolic flight campaigns with Airbus Zero-g: looking back at the A300 and looking forward to the A310. Adv. Space Res. 56, 1003-1013 (2015)

Perez-Poch, A., González, D.V., López, D.: Hypogravity research and educational parabolic flight activities conducted in barcelona: a new hub of innovation in europe. Micrograv. Sci. Technol. 28, 603-609 (2016a)

Pletser, V.: European aircraft parabolic flights for microgravity research, applications and exploration: a review. REACH 1, 11-19 (2016b)

Pletser, V., Rouquette, S., Friedrich, U., Clervoy, J.-F., Gharib, T., Gai, F., et al.: The first European parabolic flight campaign with the Airbus A310 Zero-g. Microgravity Sci. Technol. 28, 587-601 (2016c)

Pletser, V., Frischauf, N., Cohen, D., Foster, M., Spannagel, R., Szeszko, A., et al.: First middle east aircraft parabolic flights for ISU participant experiments. Micrograv. Sci. Technol. 29, 209-219 (2017a)

Pletser, V., Frischauf, N., Laufer, R., Cohen, D.: Parabolic flights with gliders as an innovative low cost platform for microgravity and hypergravity research. In: 68th International Astronautical Congress (IAC). International Astronautical Federation (IAF), Adelaide,. (IAC-17-A2-5-6-x36752) (2017b)

Pletser, V.: Gravity: Weight and Their Absence. Springer (2018)

Seibert, G., Fitton, B., Battrick, B.: A world without gravity. ESA Publications Division, Noordwijk (2001)
Selig, H., Gierse, A., König, G.: Parabolic flight with light aircraft. In: 67th International Astronautical Congress (IAC). International Astronautical Federation (IAF), Guadalajara. (IAC-16-A2-5-6x34402) (2016)

Selig, H., Gierse, A.: Migrop - parabolic flight with light aircraft a progress report. In: 68th International Astronautical Congress (IAC). International Astronautical Federation (IAF), Adelaide, Australia (IAC-17-A2-5-5-x40505) (2017)

Selig, H.: Migrop - parabolic flight with light aircraft - a new platform for zero-g, partial-g and hyper-g experiments. In: 69th International Astronautical Congress (IAC). International Astronautical Federation (IAF), Bremen. (IAC-18-A2-5-3-x43837) (2018)

Selig, H.: Migrop - parabolic flight with light aircraft - on the threshold of the market launch. In: 70th International Astronautical Congress (IAC). International Astronautical Federation (IAF), Washington. (IAC-19-A2-5-5-x53184) (2019)

Stern, G., Battelier, B., Geiger, R., Varoquaux, G., Villing, A., Moron, F., et al.: Light-pulse atom interferometry in microgravity. Eur. Phys. J. D 53, 353-357 (2009)

Thode, M., Castillo, M., Tielke, J.: Advancements in the quantification of the crystal structure of zns material produced in variable gravity. In: 42nd COSPAR Scientific Assembly. vol. 42 (2018)

Publisher's Note Springer Nature remains neutral with regard to jurisdictional claims in published maps and institutional affiliations. 